\title{
A Methodology for Obtaining the References of Voltages and Currents in Power Electronics Devices
}

\author{
Nicolás Muñoz Galeano \\ Grupo GIMEL, Departamento de Ingeniería Eléctrica, \\ Universidad de Antioquia Calle 70 No 52-21, \\ Medellín 050010, Colombia.
}

\author{
Jesús María López Lezama \\ Grupo GIMEL, Departamento de Ingeniería Eléctrica, \\ Universidad de Antioquia Calle 70 No 52-21, \\ Medellin 050010, Colombia.
}

ORCID: 0000-0002-2369-6173

ORCID: 0000-0003-1407-5559

Fernando Villada Duque

Grupo GIMEL, Departamento de Ingeniería Eléctrica, Universidad de Antioquia Calle 70 No 52-21, Medellín 050010, Colombia.

ORCID: 0000-0001-6577-2181

\begin{abstract}
This paper presents a methodology for obtaining the references for voltages and currents of power electronics (PE) devices. References are needed for deducing, in an appropriate way, the differential equations (DEs) that govern the behavior of PE devices. If references are used for deducing DEs, power and energy interchange can be explained; also, control strategies based on mathematical model can be easily implemented. PE devices are very diverse; so, the proposed methodology is explained by using a complex converter for considering many possibilities that may result. Basically, the passive law of sign is used; nonetheless, the application of this law is sometimes tricky. Finally, OpenModelica software is used for validating the proposed methodology.
\end{abstract}

Keywords: Reference voltages and currents, Power Electronics (PE) devices, Differential Equations (DE), OpenModelica.

\section{INTRODUCTION}

Deduction of reference voltages and currents are useful for deducing the DEs that govern the behavior of PE devices. The proposed methodology is a systematic procedure that takes into account diverse possibilities that can appear in PE devices. A bibliographical review shows that researches mainly focus on control strategies applied to PE devices; however, little attention is devoted to the reference setting that permits the deduction of DEs; basically, they only present DEs as a starting point and develop the control strategy. For this reason most $\mathrm{PE}$ papers are difficult to read and understand. This paper presents an understandable methodology for facilitating the comprehension of the operating principle of PE devices and the deduction of their DEs.

Using the passive law of sign, this paper develops a rigorous explanation for obtaining the references. The explanation is presented for giving specific details that can be useful to electrical, control and electronical engineers. The contributions of most power electronic design papers are related to the use of the model, failing to present details regarding the deduction of the model and the procedure for obtaining the references. However such details are of paramount importance, especially for PE designers, since references allow the understanding of the operating principle and also facilitate the design of the control structure [1]-[8].

The dynamical performance can be understood if the operation principle is explained in detail which may lead to better control strategies that fulfill the requirements established from the design [9]. There are a lot of papers that partially include the explanation of the obtention of reference voltages and currents; however, there are still many gaps in the knowledge [10]-[12]. The main contribution of this paper lies on the deduction of voltage and current references that permits a deeper explanation of the operation principle for PE devices. The proposed methodology can be easily applied to many converters [13]-[14]. After the deduction of reference voltages and currents, the proposed procedure is validated using OpenMoedlica.

OpenModelica [15] is an open source software that permits the simulation of PE devices, allowing dynamic multi-domain simulation of linear and non-linear systems. OpenModelica is conformed of an equation-based and object-oriented language (Modelica). OpenModelica incorporates extensive model libraries in many fields, a graphic connection editor (OMEdit), compiler, simulator and plotting tools. OpenModelica is widely used in industrial and research applications on electric engineering. In [16] the authors incorporate Modelica language for modelling DC microgrids. In [17] the authors simulated power electrical networks, proposing a Power System Library; also, in [18] control algorithms are implemented using this software for power inverter applications. OpenModelica is a tool for improving the formation of future engineers as indicated in [19]. In this paper, OpenModelica is used as a validation tool for verifying the obtained reference voltages and currents. 
This paper is organized as follows: Section II presents the proposed methodology using a complex converter for obtaining the reference voltages and currents. Section III corresponds to the results obtained in OpenModelica Software for validating the proposed methodology. Section IV concludes and highlights the most relevant aspect of this paper.

\section{PROPOSED METHODOLOGY}

The proposed methodology is explained using the converter depicted in Figure 1. $v_{i}$ is the input voltage; $L_{1}, L_{2}, L_{3}$, and $L_{4}$ are inductors; $C_{1}, C_{2}, C_{3}$, and $C_{4}$ are capacitors; $Q_{1}$ and $Q_{2}$ are power switches that are commutated with the same control sign; $D_{1}$ and $D_{2}$ are power diodes; and $R$ is the resistors used as a load. The following assumptions have been made: 1) Losses are neglected. 2) There drop voltage in power switches is not considered. 3) The passive law of sign is used to obtain the references of the system.

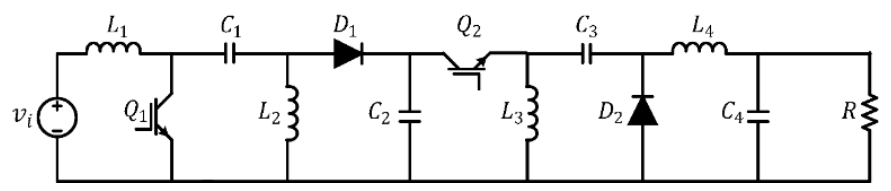

Figure 1. PE topology

The methodology consists on successively closing and opening the power switches to observe the transfer of energy between passive elements (inductors and capacitors); in this paper, each closing and opening state corresponds to a commutation. The proposed methodology is a graphical methodology in which voltage polarities and currents directions when each passive element absorbs energy are drawn. Positive voltage polarities and positive current directions are defined in this paper as the references, following the passive law sign when the element absorbs energy. While negative voltage polarities and negative current directions are defined as the reactions, under this condition the element deliver energy. After each commutation, there is only drawn the references (voltage and current references) of one passive element at a time for avoiding mistakes. References are labeled using red color while reactions are labeled using blue color. The topology of Figure 1 presents eight passive elements between inductors and capacitors; then, there are needed eight commutations. The commutations are described as follows:

Figure 2 shows the topology for the first commutation when $Q_{1}$ and $Q_{2}$ closed.

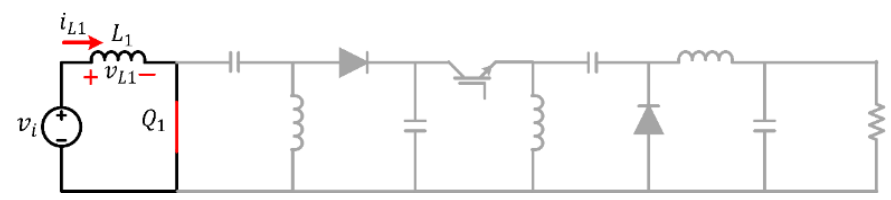

Figure 2. First commutation: $Q_{1}$ and $Q_{2}$ closed.

The first commutation allows to obtain the references for $L_{1}$. In this commutation, $v_{i}$ and $L_{1}$ are in parallel. $v_{i}$ delivers energy to $L_{1}$, so $L_{1}$ stores energy. Using the passive law of sign, it is possible to label voltage and current references (note the red voltage polarity and current direction). Also, it is recommended to label one reference at a time for avoiding mistakes.

Figure 3 shows the topology for the second commutation when $Q_{1}$ and $Q_{2}$ are open.

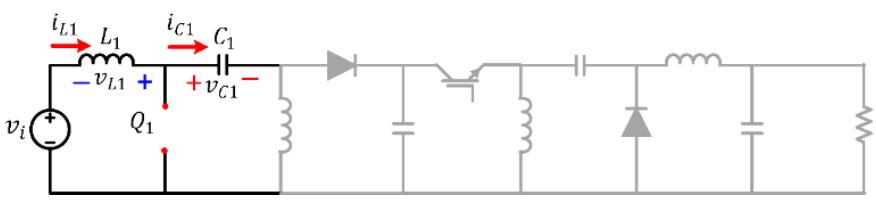

Figure 3. Second commutation: $Q_{1}$ and $Q_{2}$ open.

The second commutation permits the obtention of references for $C_{1}$. In this commutation, $L_{1}$ reacts changing its voltage polarity. Voltage reaction of $L_{1}$ is labeled using blue color. Then, $v_{i}$ and $L_{1}$ are in parallel with $C_{1}$, both $v_{i}$ and $L_{1}$ deliver energy to $C_{1}$. According to the passive law of sign, it is possible to label voltage and current references for $C_{1}$ using red color.

Figure 4 shows the topology for the third commutation when $Q_{1}$ and $Q_{2}$ are closed again.

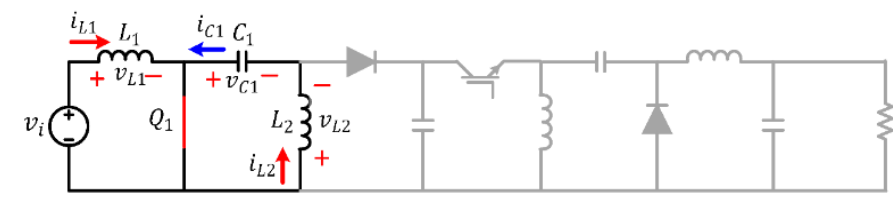

Figure 4. Third commutation: $Q_{1}$ and $Q_{2}$ closed.

The third commutation permits the obtention of references for $L_{2}$. In this commutation, $C_{1}$ reacts changing its current direction. Current reaction of $C_{1}$ is labeled using blue color. Then, $v_{i}$ and $L_{1}$ are in parallel with $C_{1}$, both $v_{i}$ and $L_{1}$ deliver energy to $C_{1}$. According to the passive law of sign, it is possible to label voltage and current references for $C_{1}$ using red color.

Figure 5 shows the topology for the fourth commutation when $Q_{1}$ and $Q_{2}$ are open again.

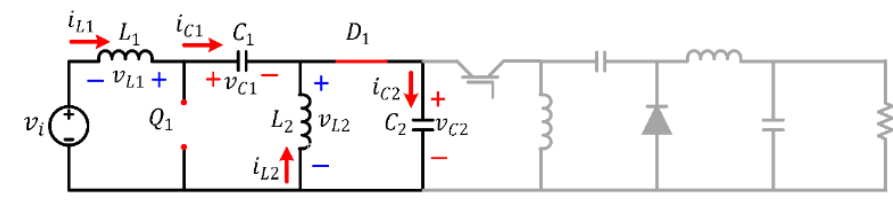

Figure 5. Fourth commutation: $Q_{1}$ and $Q_{2}$ open.

The fourth commutation permits the obtention of references for $C_{2}$. In this commutation, $L_{2}$ reacts changing its voltage polarity. Voltage reaction of $L_{2}$ is labeled using blue color. Voltage reaction directly polarized $D_{1}$; so, $L_{2}$ is in parallel with $C_{2} . L_{2}$ delivers its energy to $C_{2}$. According to the passive law of sign, it is possible to label voltage and current references for $C_{2}$ using red color. 
Figure 6 shows the topology for the fifth commutation when $Q_{1}$ and $Q_{2}$ are closed again.

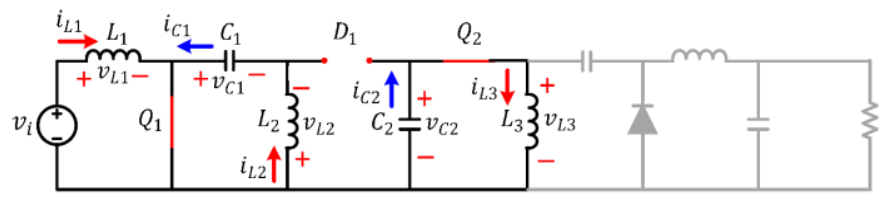

Figure 6. Fifth commutation: $Q_{1}$ and $Q_{2}$ closed

The fifth commutation permits the obtention of references for $L_{3}$. In this commutation, $C_{2}$ reacts changing its current direction. Current reaction of $C_{2}$ is labeled using blue color. In this commutation, $C_{2}$ is in parallel with $L_{3} . C_{2}$ delivers its energy to $L_{3}$. According to the passive law of sign, it is possible to label voltage and current references for $L_{3}$ using red color.

Figure 7 shows the topology for the sixth commutation when $Q_{1}$ and $Q_{2}$ are open again.

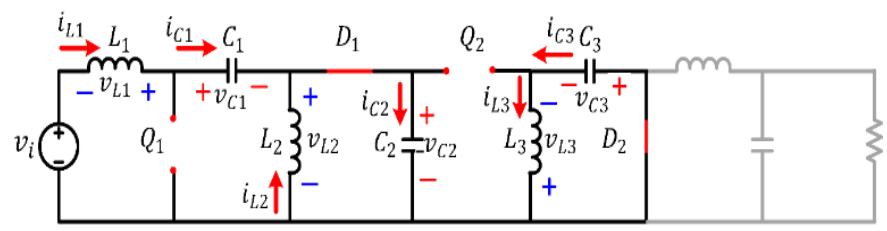

Figure 7. Sixth commutation: $Q_{1}$ and $Q_{2}$ open.

The sixth commutation permits the obtention of references for $C_{3}$. In this commutation, $L_{3}$ reacts changing its voltage polarity. Voltage reaction of $L_{3}$ is labeled using blue color. Voltage reaction directly polarized $D_{2} ;$ so, $L_{3}$ is in parallel with $C_{3}$. $L_{3}$ delivers its energy to $C_{3}$. According to the passive law of sign, it is possible to label voltage and current references for $C_{3}$ using red color.

Figure 8 shows the topology for the seventh commutation when $Q_{1}$ and $Q_{2}$ are closed again.

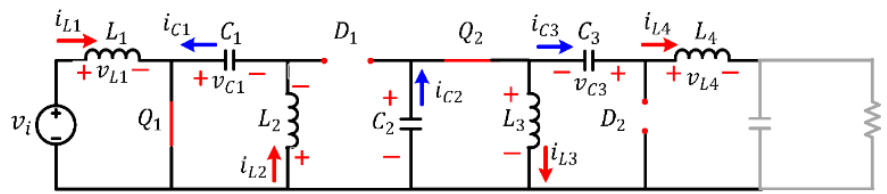

Figure 8. Seventh commutation: $Q_{1}$ and $Q_{2}$ closed.

The seventh commutation permits the obtention of references for $L_{4}$. In this commutation, $C_{3}$ reacts changing its current direction. Current reaction of $C_{3}$ is labeled using blue color. $C_{3}$ delivers energy to $L_{4}$. According to the passive law of sign, it is possible to label voltage and current references for $L_{4}$ using red color.
Figure 9 shows the topology for the eighth commutation when $Q_{1}$ and $Q_{2}$ are open again.

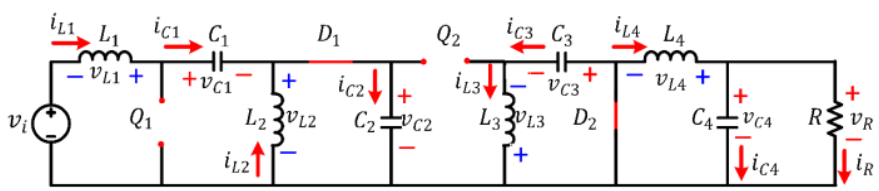

Figure 9. Eighth commutation: $Q_{1}$ and $Q_{2}$ open.

The eighth commutation permits the obtention of references for $C_{4}$. In this commutation, $L_{4}$ reacts changing its voltage polarity. Voltage reaction of $L_{4}$ is labeled using blue color. $L_{4}$ are in parallel with $C_{4}$ and $R . L_{4}$ delivers its energy to $C_{4}$ and $R$. According to the passive law of sign, it is possible to label voltage and current references for $C_{4}$ using red color.

Figure 10 shows the topology for the nineth commutation when $Q_{1}$ and $Q_{2}$ are closed again.

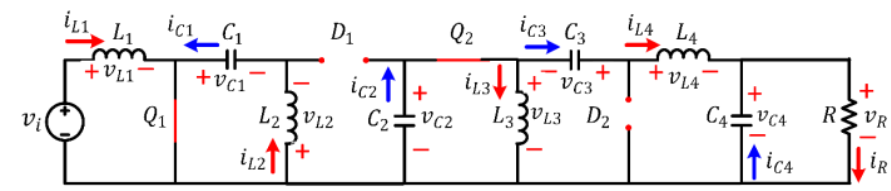

Figure 10. Nineth commutation: $Q_{1}$ and $Q_{2}$ open.

The nineth commutation permits verifying if there is an appropriate transfer of energy in the converter. So, $C_{4}$ reacts changing its current direction. Current reaction of $C_{4}$ is labeled using blue color. It is confirmed that $C_{4}$ delivers its energy to $R$.

Figure 11 show the summary of references that must be used for obtaining the set of DEs that govern the behavior of the converter.

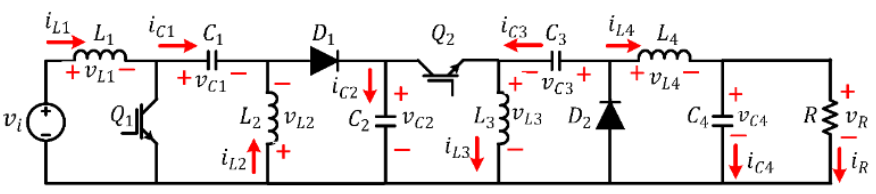

Figure 11. Summary voltage and current references.

\section{RESULTS}

Simulation was carried out using the default compiler and solver in OpenModelica connection editor (OMEdit), version 3.2.2. Figure 12 show the implementation in OpenModelica of converter depicted in Figure 1. Duty cycle used was $20 \%$ while switching frequency was $1 \mathrm{kHz}$. The obtained voltage and current references were used for the implementation. In OpenModelica for each element, the squares filled with blue correspond to the "+" signs of the references, while the squares that are not filled correspond to the "_." signs of the references. 


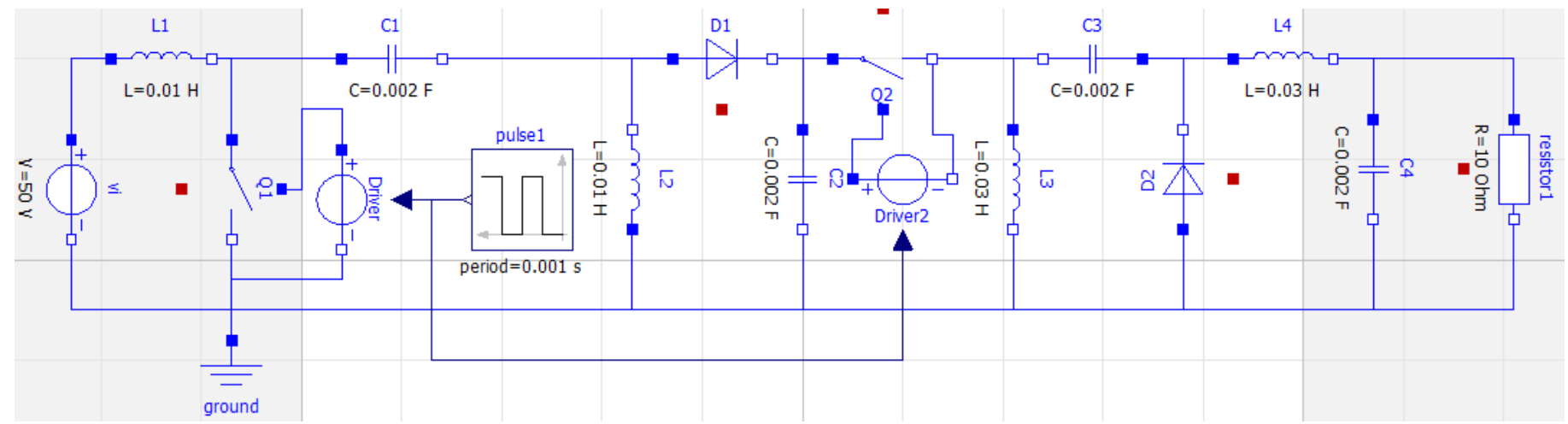

Figure 12. Implementation in OpenModelica.

Figure 13 shows all waveforms that result after the simulation. At the bottom of the Figure, there are depicted PWM (Pulse Width Modulation) signal for all columns of figures. In each switching state, there are confirmed the obtained voltage and current references when waveforms are positive, while reactions are also confirmed when waveforms are negative. It is concluded through simulation that the proposed methodology correctly determines the voltage and current references of the converter.
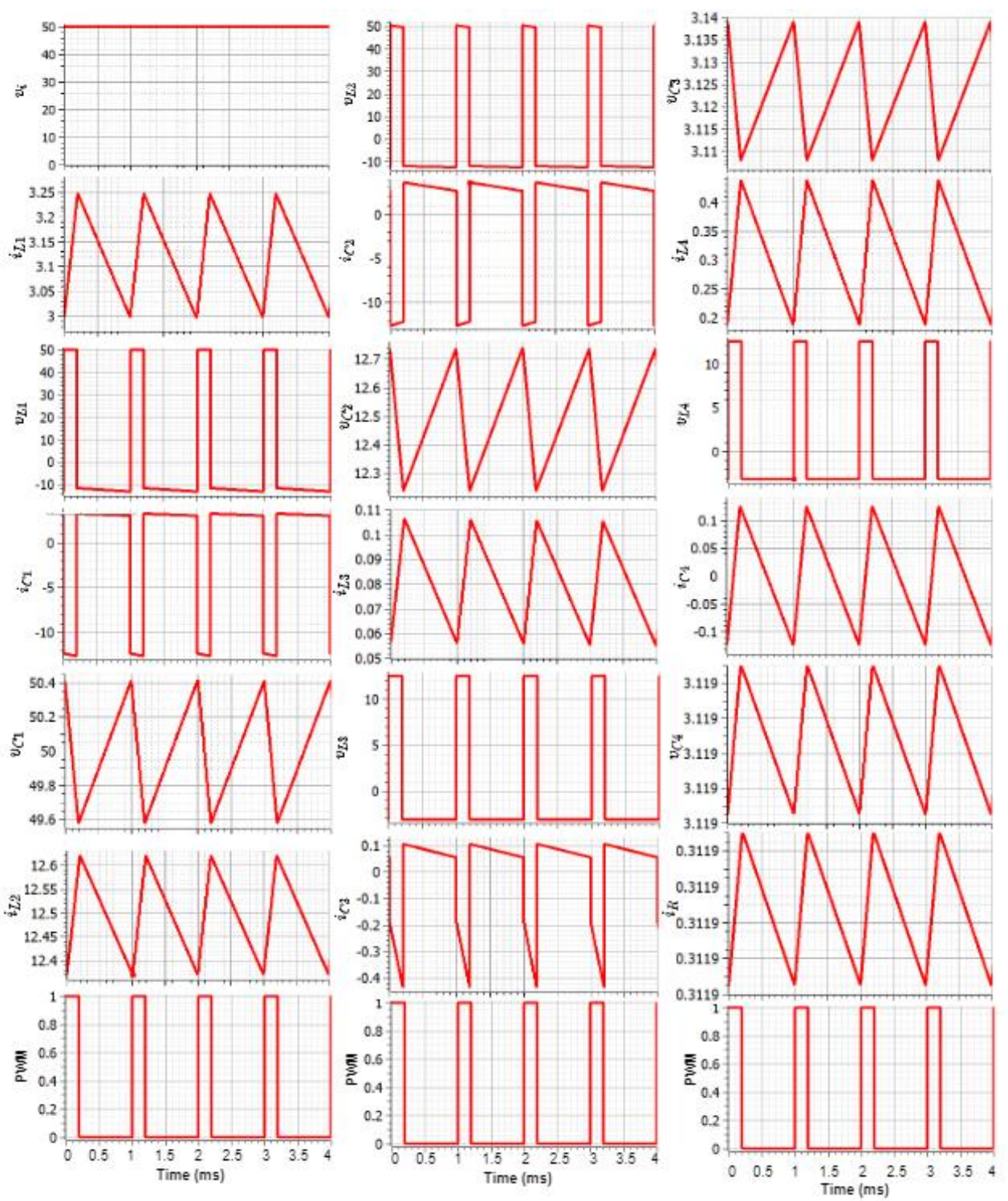

Figure 13. Voltage and current waveforms of the converter. 


\section{CONCLUSIONS}

This paper presented a methodology for obtaining voltage and current references that permits the deduction of DEs that govern the behavior of PE converters. Methodology was based on the passive law of sign. The application of this law was explained using a converter that considers diverse possibilities that could results in PE field. The proposed methodology consists of successively closing and opening the power switches to observe the transfer of energy between passive elements. Basically, the methodology is a graphical methodology in which voltage polarities and currents directions when each passive element absorbs energy are drawn. Methodology was validated through the implementation of the converter in OpenModelica software, it is concluded that the proposed methodology correctly determines the voltage and current references for PE converters.

\section{Acknowledgements}

The authors gratefully acknowledge the financial support provided by the Colombia Scientific Program within the framework of the call Ecosistema Científico (Contract No. FP44842- 218-2018). Likewise, Universidad de Antioquia (Colombia) is acknowledged for the financial support through the Sostenibilidad program.

\section{REFERENCES}

[1] Arango, E., Ramos-Paja, C. A., Calvente, J., Giral, R., and Serna-Garces, S. I. (2013). Asymmetrical Interleaved DC/DC Switching Converters for Photovoltaic and Fuel Cell Applications - Part 2: Control-Oriented Models. Energies, 6(10), 5570-5596.

[2] Bi, Z. and Xia, W. (2010). Modeling and Simulation of Dual-Mode DC/DC Buck Converter. Second International Conference on Computer Modeling and Simulation, Sanya, Hainan (China), 371-375.

[3] Urrea-Quintero, JH., Muñoz-Galeano, N., GómezEchavarría, LM. (2018). Analysis and Control of Power Electronic Converters Based on a System Zeros Location Approach. In Anh Tuan, L. Applied Modern Control (pp.122). London: Intechopen.

[4] Duong, T-D., Nguyen, M-K., Tran, T-T., Lim, Y-C., Choi, J-H. (2019). Transformerless High Step-Up DC-DC Converters with Switched-Capacitor Network. Electronics, 8(12), 1420.

[5] Martínez-García, MS., de Castro, A., Sanchez, A., Garrido, J. (2019). Analysis of Resolution in Feedback Signals for Hardware-in-the-Loop Models of Power Converters. Electronics, 8(12), 1527.

[6] Shaw, P. (2019). Modelling and analysis of an analogue MPPT-based PV battery charging system utilising dc-dc boost converter. IET Renewable Power Generation, 13(11), 1958-1967
[7] Velilla, E., Cano, J. B., Jaramillo, F. (2019). Monitoring system to evaluate the outdoor performance of solar devices considering the power rating conditions. Solar Energy, 194(1), $79-85$

[8] Yang, T., Liao, Y. Discrete Sliding Mode Control Strategy for Start-Up and Steady-State of Boost Converter. (2019). Energies, 12(15), 2990.

[9] Liu, J., Hu, J. and Xu, L. (2007). Dynamic Modeling and Analysis of Z Source Converter -- Derivation of AC Small Signal Model and Design-Oriented Analysis. Power Electron. IEEE Trans., 22(5), 1786-1796.

[10] Beldjajev, V. and Roasto, I. (2012). Efficiency and Voltage Characteristics of the Bi-Directional Current Doubler Rectifier. Przeglad Elektrotechniczny, 88(8), 124-129.

[11] Rouzbehi, K., Miranian, A., Escaño, JM., Rakhshani, E., Shariati, N., Pouresmaeil, E. (2019). A Data-Driven Based Voltage Control Strategy for DC-DC Converters: Application to DC Microgrid. Electronics, 8(5), 493.

[12] Davoudi, A., Jatskevich, J., Chapman, P. L. and Bidram, A. (2013). Multi-Resolution Modeling of Power Electronics Circuits Using Model-Order Reduction Techniques. IEEE Transactions on Circuits and Systems I: Regular Papers, 60(3), 810-823.

[13] Geyer, T., Papafotiou, G., Frasca, R. and Morari, M. (2008). Constrained Optimal Control of the Step-Down DCDC Converter. IEEE Transactions on Power Electronics, 23(5), 2454-2464.

Liang, T. J. and Tseng, K. C. (2005). Analysis of integrated boost-flyback step-up converter. IEE Proceedings - Electric Power Applications, 152(2), 217-225.

[14] Restrepo, C., Konjedic, T., Calvente, J., Milanovic, M. and Giral, R. (2013). Fast Transitions Between Current Control Loops of the Coupled-Inductor Buck-Boost DC-DC Switching Converter. IEEE Transactions on Power Electronics, 28(8), 3648-3652.

[15] Fritzson, P., Pop, A., Asghar, A., Bachmann, B., Braun, W., Braun, R., ... , Franke, R. (october, 2019). The OpenModelica Integrated Modeling, Simulation, and Optimization Environment. In Proceedings of The American Modelica Conference 2018, Somberg Conference Center, Cambridge MA, USA, Linköping University Electronic Press.

[16] Dizqah, A. M., Maheri, A., Busawon, K., Fritzson, P. (2015). Standalone DC microgrids as complementarity dynamical systems: Modeling and applications. Control Engineering Practice, 35, 102-112.

[17] Bartolini, A., Casella, F., Guironnet, A. (march, 2019). Towards Pan-European Power Grid Modelling in Modelica: Design Principles and a Prototype for a Reference Power System Library. In Proceedings of the 13th International Modelica Conference, Regensburg (Germany), Linköping University Electronic Press.

[18] Reid, D. (april, 2015). DQ rotating frame PI control algorithm for power inverter voltage regulation modelling and simulation using the OpenModelica platform. In 
International Journal of Engineering Research and Technology. ISSN 0974-3154, Volume 13, Number 11 (2020), pp. $3272-3277$

(C) International Research Publication House. https://dx.doi.org/10.37624/IJERT/13.11.2020.3272-3277

SoutheastCon 2015, Fort Lauderdale (EEUU), IEEE.

[19] Murad, M. A. A., Vanfretti, L., Rokonuzzaman, M.,

Tuhin, R. A. (september, 2017). Enhancing engineering studies in developing countries using OpenModelica. In 2017 4th International Conference on Advances in Electrical Engineering, Dhaka (Bangladesh) 